\title{
INDEX ESTIMATES FOR FREE BOUNDARY CONSTANT MEAN CURVATURE SURFACES
}

\author{
MARCOS P. CAVALCANTE AND DARLAN F. DE OLIVEIRA

\begin{abstract}
In this paper we consider compact constant mean curvature surfaces with boundary immersed in a mean convex body of the Euclidean space or in the unit sphere. We prove that the Morse index is bounded from below by a linear function of the genus and number of boundary components.
\end{abstract}

\section{INTRODUCTION}

Let $W$ be a Riemannian manifold with nonempty boundary such that its boundary $\partial W$ is a union of smooth hypersurfaces. Let $M \subset$ $W$ be a compact constant mean curvature hypersurface such that $M$ intersects the regular part of $\partial W$ along its boundary in a right angle. It is well known that such hypersurfaces are critical points of the area functional for variations of $M$ that preserve the enclosed volume and keep the boundary freely on $\partial W$. These hypersurfaces arise in many geometrical and physical problems and are referred as free boundary CMC hypersurfaces (FBCMC hypersurfaces, for short). They have been studied since the 19th century and still now form a very active topic in differential geometry. We refer the reader to the books of Finn [7] and López [13], as well as the references therein, for a nice introduction to this subject.

An important problem about FBCMC hypersurfaces is to classify those ones that are stable, that is, whose the second variation of the area is nonnegative for volume preserving variations. For instance, in the case that $W$ is a geodesic ball in a space form, a well-known conjecture asserted that spherical caps are the only solutions. It was confirmed by the works of Ros-Vergasta 21] and Nunes [16] in dimension two and more recently by Wang and Xia [24] in any dimension. Other results

Date: March 19, 2018.

2010 Mathematics Subject Classification. 53A10, 49Q10, 35P15.

Key words and phrases. Constant mean curvature surfaces, Morse index, free boundary surfaces.

The first author was partially supported by CNPq-Brazil. 
on stable FBCMC hypersurfaces can be found for instance in [1], 3], [4], 6], [11], [12], [14], [17] and [20].

When $M$ is a stable FBCMC surface immersed in a mean convex region $W \subset \mathbb{R}^{3}$ Ros showed in [17, Theorem 9] that there are just a few possibilities for the genus and the number of boundary components of $M$ (see Corollary 1.2 below).

When $M$ is not stable, the Jacobi operator $J$ associated to the second variation of the area has non zero Morse index (see Section 2 for precise definitions). Geometrically, the index of $M$ is the number of directions whose admissible variations decrease area. It will be denoted by $\operatorname{Ind}_{\mathrm{w}}(M)$.

The case of free boundary minimal surfaces is of special interest and the works of Fraser and Schoen [8, 9, 10] have motivated many research in this case. For free boundary minimal hypersurfaces all variations keeping the boundary freely in the boundary are allowed, not only volume preserving variations. In this setting, recently Ambrozio, Carlotto and Sharp in [2] and Sargent in [22], proved independently that if $M$ is a free boundary minimal surface immersed in a mean convex region $W \subset \mathbb{R}^{3}$ then the index is bounded from below by $(2 g+$ $k-1) / 3$, where $g$ is the genus of $M$ and $k$ is the number of boundary components. In higher dimensions, they also obtained lower bounds for the index in terms of the dimension of the first relative homology group with real coefficients. The technique presented in these results uses the coordinates of harmonic forms as test functions and is inspired by previous works on eigenvalue estimates and index estimates for minimal hypersurfaces without boundary (see [18, 23] and [2]).

Following these lines, in this paper, we obtain a comparison theorem for the eigenvalues of $J$ in terms of the mean curvature and the eigenvalues of the Hodge Laplacian $\Delta$ acting on 1-forms and, as a by-product, we obtain lower bounds for the Morse index of FBCMC surfaces in mean convex regions of the Euclidean space or the unit sphere. More precisely, our results are the following.

Theorem 1.1. Let $W$ be a region of $\mathbb{R}^{3}$ such that its boundary is a union of smooth mean convex surfaces, and let $M^{2}$ be a compact, orientable, FBCMC surface immersed in the mean convex side of $W$ and whose boundary intersects the regular part of $\partial W$. Then for all positive integers $\alpha$ we have

$$
\lambda_{\alpha}^{J} \leq-2 H^{2}+\lambda_{m(\alpha)}^{\Delta}
$$

where $m(\alpha)>6(\alpha-1)$. 
In particular, if $M$ has genus $g$ and $k$ boundary components. Then,

$$
\operatorname{Ind}_{\mathrm{w}}(M) \geq \frac{2 g+k-4}{6} .
$$

As an immediate consequence we obtain the result of Ros [17] cited above.

Corollary 1.2 (Ros). Under the conditions of Theorem 1.1, if $M$ is stable, then only possibilities for $g$ and $k$ are

(1) $g=0$ and $k \leq 4$;

(2) $g=1$ and $k=1$ or 2 .

In the case of FBCMC surfaces immersed in mean convex domains of $\mathbb{S}^{3}$, the result read as follows.

Theorem 1.3. Let $W$ be a region in the unit sphere $\mathbb{S}^{3}$ such that its boundary is a union of smooth mean convex surfaces, and let $M^{2}$ be a compact, orientable, FBCMC surface immersed in the mean convex side of $W$ and whose boundary intersects the regular part of $\partial W$. Then for all positive integers $\alpha$ we have

$$
\lambda_{\alpha}^{J} \leq-2\left(H^{2}+1\right)+\lambda_{m(\alpha)}^{\Delta},
$$

where $m(\alpha)>8(\alpha-1)$.

In particular,

$$
\operatorname{Ind}_{\mathrm{w}}(M) \geq \frac{2 g+k-5}{8} .
$$

Corollary 1.4. Under the conditions of Theorem 1.3, if $M$ is stable, then only possibilities for $g$ and $k$ are

(1) $g=0$ and $k \leq 4$;

(2) $g=1$ and $k=1$ or 2 ;

(3) $g=2$ and $k=1$.

This paper is organized as follows. In Section 2 we present somen definitions and basic results to be used in the proofs. Section 3 is devoted to computing the Jacobi operator of the test functions given by the coordinates of harmonic forms. In Section 4 we present the proof of Theorem 1.1. The proof of Theorem [1.3 is analogous and will be omitted.

\section{Preliminaries}

Let us denote by $W$ a domain of the Euclidean space $\mathbb{R}^{3}$ not necessarily compact. For simplicity, let us assume that $W$ has smooth 
boundary and let $\nu$ be the unit normal vector field along $\partial W$. We recall that the second fundamental form and the mean curvature of $\partial W$ with respect to $\nu$ are defined respectively by

$$
I I^{\partial W}(X, Y)=\left\langle-D_{X} \nu, Y\right\rangle, \quad \text { for } X, Y \in T \partial W,
$$

and

$$
H^{\partial W}=\frac{1}{2} \operatorname{tr} I I^{\partial W}
$$

where $D$ is the Levi-Cevita connection in the Euclidean space. If we can choose the unit normal $\nu$ along $\partial W$ such that $\nu$ points outward $W$ and $I I^{\partial W}$ is negative defined, then we say that $W$ is convex. If it can be chosen such that $H^{\partial W} \leq 0$, then we say that $W$ is mean convex.

Let $x: M \rightarrow W$ be a compact oriented surfaced with boundary which is properly immersed, that is, $M \cap W=\partial M$. Fixed a unit normal vector field $N$ along $x$ we denote by $A$ the shape operator associated to the second fundamental form $I I^{\partial M}$ of $M$ with respect to $N$, namelly

$$
A X=-D_{X} N, \quad \text { for } X \in T M
$$

and

$$
I I^{\partial M}(X, Y)=\langle A X, Y\rangle, \quad \text { for } X, Y \in T M,
$$

We say that $M$ is free boundary if $\partial M$ meets $\partial W$ orthogonally.

From now on, let us assume that $W$ is a mean convex domain of $\mathbb{R}^{3}$ and $M$ is a free boundary constant mean curvature surface properly immersed in $W$. Such surfaces are critical points of the area functional for variations that preserves the enclosed volume (see [15]). It is easy to see that, up a parametrization, we can consider only normal variations, say generated by $X=u N$, where $u \in \mathcal{F}$ and

$\mathcal{F}=\left\{u: M \rightarrow \mathbb{R}: u\right.$ is smooth up to the boundary and $\left.\int_{M} u d M=0\right\}$.

In this setting, the second variation of area functional is given by the following quadratic form (see [21], [17])

$$
Q(u, u)=\int_{M}\left(u \Delta u-\|A\|^{2} u^{2}\right) d M+\int_{\partial M}\left(u \eta(u)+I I^{\partial W}(N, N) u^{2}\right) d s .
$$

Here $\eta$ is the unit conormal vector to $M$ along its boundary. Note that the free boundary condition means that $\eta=\nu$ along $\partial W$. We point out that in this paper we are using the geometric definition of the Laplacian operator, that is, $\Delta u=\operatorname{div} \nabla u$, where $\operatorname{div} X=-\operatorname{tr} \nabla X$.

The index of $M$, denoted by $\operatorname{Ind}_{\mathrm{w}}(M)$, is defined as the maximal dimension of a subspace of $\mathcal{F}$ on which $Q$ is negative. Geometrically, the index indicates the number of directions whose variations decrease area. In particular, we say that $M$ is stable if the index is zero. We 
also can define the index as the number of negative eigenvalues of the boundary problem associated to the quadratic form $Q$. More precisely, let us consider $J=\Delta-\|A\|^{2}$ the Jacobi operator of $M$. We say that $\lambda^{J} \in \mathbb{R}$ is an eigenvalue of $Q$ if there exists a nontrivial eigenfunction $u \in \mathcal{F}$ such that

$$
\left\{\begin{array}{l}
J u=\lambda^{J} u, \quad \text { in } M, \\
\frac{\partial u}{\partial \eta}=-I I^{\partial B}(N, N) u, \quad \text { on } \partial M .
\end{array}\right.
$$

It is well known that there exists a non-decreasing sequence

$$
\lambda_{1}<\lambda_{2} \leq \cdots \leq \lambda_{k} \leq \cdots \nearrow \infty
$$

of eigenvalues associated to a $L^{2}(M)$-orthonormal basis $\left\{\phi_{1}, \cdots, \phi_{k}, \cdots\right\}$ of solutions to the eigenvalue problem (2.1) and satisfying the min-max characterization

$$
\lambda_{k}^{J}=\min _{u \in \mathcal{G}} \frac{Q(u, u)}{\int_{M} u^{2} d M},
$$

where $\mathcal{G}=\left\langle\phi_{1}, \cdots, \phi_{k-1}\right\rangle^{\perp} \backslash\{0\}$. It follows immediately that $\operatorname{Ind}_{\mathrm{w}}(M)$ equals the number of negative eigenvalues of the problem (2.1).

In order to give lower bounds for the index of $M$ in terms of its topological invariants we will construct admissible eigenfunctions in $\mathcal{F}$ using harmonic vector fields, or equivalently harmonic 1-forms. Let us consider the set of 1 -forms that are normal at $\partial M$, that is,

$$
\Omega^{1}(M, \partial M):=\left\{w \in \Omega^{1}(M), i^{*} w=0\right\},
$$

where $i: \partial M \rightarrow M$ is the inclusion map.

We then consider the space of tangential harmonic 1-forms

$$
\mathcal{H}_{N}^{1}(M):=\left\{w \in \Omega^{1}(M, \partial M) ; d w=0 \text { and } \delta w=0\right\}
$$

and the space of normal harmonic 1-forms

$$
\mathcal{H}_{T}^{1}(M):=\left\{\star w \in \Omega^{1}(M, \partial M) ; d w=0 \text { and } \delta w=0\right\} .
$$

Above $d$ is the exterior derivative operator and $\delta$ is the interior derivative operator defined by $\delta=-\star d \star$, where $\star: \Omega^{1}(M) \rightarrow \Omega^{1}(M)$ is the Hodge star operator.

It is well known that these spaces are closed related to the topology of the underline manifold. In fact we have the following result (see [2] or $[22]$ ).

Lemma 2.1. Let $M^{2}$ be a compact, orientable surface with non-empty boundary $\partial M$. If $M$ has genus $g$ and $k \geq 1$ boundary components, then

$$
\operatorname{dim} \mathcal{H}_{T}^{1}(M)=\operatorname{dim} \mathcal{H}_{N}^{1}(M)=2 g+k-1 .
$$




\section{TEST FUnCTIONS AND HARMONIC VECTOR FIELDS}

Denoting by $\mathcal{E}=\left\{\bar{E}_{1}, \bar{E}_{2}, \bar{E}_{3}\right\}$ the canonical basis in $\mathbb{R}^{3}$ we will considere $E_{i}:=\bar{E}_{i}-\left\langle\bar{E}_{i}, N\right\rangle N$, the orthogonal projection of $\bar{E}_{i}$ on $T M$. We also consider the smooth support functions $g_{i}: M \rightarrow \mathbb{R}$, $g_{i}:=\left\langle\bar{E}_{i}, N\right\rangle$, for $1 \leq i \leq 3$.

Given a smooth vector field $\xi \in T M$ on $M$ we will use its coordinates and the coordinates of $\star \xi$ as test functions. Namely, for each $1 \leq i \leq 3$, we define $w_{i}, \bar{w}_{i}: M \rightarrow \mathbb{R}$ as

$$
w_{i}:=\left\langle E_{i}, \xi\right\rangle \text { and } \bar{w}_{i}:=\left\langle E_{i}, \star \xi\right\rangle .
$$

In order to compute the Jacobi operator of $w_{i}$ and $\bar{w}_{i}$ we recall the following lemma of local nature proved in [5] (see also [19]).

Lemma 3.1. Let $M^{2}$ be an orientable CMC surface in $\mathbb{R}^{3}$. Then, using the above notation we have

$$
\Delta w_{i}=\left(\|A\|^{2}-4 H^{2}\right) w_{i}+2 H\left\langle A E_{i}, \xi\right\rangle-2 g_{i}\langle A, \nabla \xi\rangle+\left\langle E_{i}, \Delta \xi\right\rangle,
$$

and

$$
\Delta \bar{w}_{i}=\left(\|A\|^{2}-4 H^{2}\right) \bar{w}_{i}+2 H\left\langle A E_{i}, \star \xi\right\rangle-2 g_{i}\langle A, \nabla \star \xi\rangle+\left\langle E_{i}, \Delta \star \xi\right\rangle,
$$

for $1 \leq i \leq 3$.

Now we note that when the vector field $\xi$ is harmonic and tangential along $\partial M$ its coordinates are admissible functions to compute the index of CMC surfaces. More precisely we have:

Lemma 3.2. If $\xi \in T M$ is a harmonic vector field which is tangential in $\partial M$, then $w_{i} \in \mathcal{F}$, that is,

$$
\int_{M} w_{i} d M=0
$$

for $1 \leq i \leq 3$.

Proof. Note that $E_{i}=\nabla x_{i}, 1 \leq i \leq 3$, where $x=\left(x_{1}, x_{2}, x_{3}\right): M \rightarrow B$ is the immersion map. Then we have

$$
\begin{aligned}
\int_{M} w_{i} d M & =\int_{M}\left\langle\nabla x_{i}, \xi\right\rangle d M \\
& =\int_{M} x_{i} \operatorname{div} \xi d M+\int_{\partial M} x_{i}\langle\xi, \eta\rangle d s=0 .
\end{aligned}
$$

In fact, $\operatorname{div} \xi=0$ since $\xi$ is harmonic and $\langle\xi, \eta\rangle=0$, since $\xi$ tangential to $\partial M$. 
Remark 3.3. In general the functions $\bar{w}_{i}, 1 \leq i \leq 3$, have not mean value zero. However, we will see in Section 4 that if $\operatorname{dim} \mathcal{H}_{T}^{1}(M)$ is large enough then we can choose $\xi$ such that

$$
\int_{M} \bar{w}_{i} d M=0
$$

for $1 \leq i \leq 3$.

We conclude this section computing the boundary term of the quadratic form $Q$ on $w_{i}$ and $\bar{w}_{i}$.

Lemma 3.4. If $\xi \in T M$ is a harmonic vector field which is tangential in $\partial M$, then

$$
\sum_{i} \int_{\partial M}\left(w_{i} \eta\left(w_{i}\right)+I I^{\partial W}(N, N) w_{i}^{2}\right) d s=2 \int_{\partial M} H^{\partial W}\|\xi\|^{2} d s
$$

and

$$
\sum_{i} \int_{\partial M}\left(\bar{w}_{i} \eta\left(\bar{w}_{i}\right)+I I^{\partial W}(N, N) \bar{w}_{i}^{2}\right) d s=2 \int_{\partial M} H^{\partial W}\|\xi\|^{2} d s .
$$

Proof. We first note that for any vector field $X$ we have

$$
\begin{aligned}
\left\langle\nabla_{\eta} E_{i}, X\right\rangle & =\eta\left\langle\bar{E}_{i}, X\right\rangle-\left\langle\bar{E}_{i}, \nabla_{\eta} X\right\rangle \\
& =\left\langle\bar{E}_{i}, D_{\eta} X-\nabla_{\eta} X\right\rangle \\
& =\left\langle\bar{E}_{i}, N\right\rangle\langle\xi, A \eta\rangle .
\end{aligned}
$$

For $\xi$ tangential at $\partial M$ we have

$$
\begin{aligned}
\sum_{i} \int_{\partial M} w_{i} \eta\left(w_{i}\right) d s & =\sum_{i} \int_{\partial M} w_{i}\left(\left\langle\nabla_{\eta} \xi, E_{i}\right\rangle+\left\langle\bar{E}_{i}, N\right\rangle\langle\xi, A \eta\rangle\right) d s \\
& =\int_{\partial M}\left\langle\nabla_{\eta} \xi, \xi\right\rangle d s \\
& =\int_{\partial M}\left\langle\nabla_{\xi} \xi, \eta\right\rangle d s \\
& =-\int_{\partial M}\left\langle\nabla_{\xi} \eta, \xi\right\rangle d s \\
& =\int_{\partial M} I I^{\partial W}(\xi, \xi) d s .
\end{aligned}
$$

Since $\xi$ and $N$ form an orthogonal basis of the tangent space of $\partial W$ along $\partial M$ we conclude the proof by noting that

$$
I I^{\partial W}(\xi, \xi)+I I^{\partial W}(N, N)\|\xi\|^{2}=2 H^{\partial W}\|\xi\|^{2} .
$$


The proof of assertion (3.2) follows the same steps above noting additionally that the Levi-Civita connection $\nabla$ commutes with the Hodge star operator $\star$.

\section{Proof of Theorem 1.1}

The proofs follow the same spirit as we did in [5] but taking in account the boundary term. We point out here that we can assume without loss of generality that $\partial W$ is smooth. Let $\xi_{1}, \xi_{2}, \ldots, \xi_{m}$ be the first $m$ eigenfunctions of the Hodge Laplacian $\Delta$ and set $\mathcal{L}_{m}^{\Delta}=$ $\operatorname{span}\left\{\xi_{1}, \ldots, \xi_{m}\right\}$ the vector space generated by these functions. Clearly, $\mathcal{H}_{T}^{1}(M)$ is a subspace of $\mathcal{L}_{m}^{\Delta}$, and by Lemma $2.1 \operatorname{dim} \mathcal{H}_{T}^{1}(M)=2 g+k-1$.

Next, we set an orthonormal basis of $C^{\infty}(M)$ given by eigenfunctions of the Jacobi operator, say $\left\{\phi_{1}, \phi_{2}, \ldots\right\}$. We denote by $\mathcal{J}^{p}:=$ $\left\langle\phi_{1}, \cdots, \phi_{p}\right\rangle^{\perp}$ the linear space orthogonal to the first $p$ eigenfunctions of the Jacobi operator.

Initially, we look for vector fields $\xi \in \mathcal{L}_{m}^{\Delta}$ such that the functions $w_{i}, \bar{w}_{i} \in \mathcal{J}^{\alpha-1}$, for some $\alpha \in \mathbb{N}$ and $i \in\{1,2,3\}$. It is equivalent to find a solution to the following system with $6(\alpha-1)$ homogenous linear equations in the variable $\xi$

$$
\int_{M} w_{i} \phi_{k} d M=\int_{M} \bar{w}_{i} \phi_{k} d M=0,
$$

$1 \leq i \leq 3$ and $1 \leq k \leq \alpha-1$. In particular, if $m(\alpha):=\operatorname{dim} \mathcal{L}_{m}^{\Delta}>$ $6(\alpha-1)$, then the system (4.1) has at least one non trivial solution $\xi \in \mathcal{L}_{m}^{\Delta}$ such that $w_{i}, \bar{w}_{i} \in \mathcal{J}^{\alpha-1}$ for all $1 \leq i \leq 3$. By Courant minimax principle we have

$$
\lambda_{\alpha}^{J} \int_{M} w_{i}^{2} d M \leq Q\left(w_{i}, w_{i}\right) \quad \text { and } \quad \lambda_{\alpha}^{J} \int_{M} \bar{w}_{i}^{2} d M \leq Q\left(\bar{w}_{i}, \bar{w}_{i}\right) .
$$

Now, using Lemma 3.1 we get

$$
\begin{aligned}
\lambda_{\alpha}^{J} \int_{M} w_{i}^{2} d M \leq & -4 H^{2} \int_{M} w_{i}^{2} d M+2 H \int_{M}\left\langle E_{i}, A \xi\right\rangle w_{i} d M \\
& +\int_{M}\left\langle E_{i}, \Delta \xi\right\rangle w_{i} d M-2 \int_{M} g_{i}\langle A, \nabla \xi\rangle w_{i} d M \\
& +\int_{\partial M}\left(w_{i} \eta\left(w_{i}\right)+I I^{\partial W(N, N)} w_{i}^{2}\right) d s .
\end{aligned}
$$


Summing up $i=1,2,3$ and using Lemma 3.4 we obtain

$$
\begin{aligned}
\lambda_{\alpha}^{J} \int_{M}\|\xi\|^{2} d M \leq & -4 H^{2} \int_{M}\|\xi\|^{2} d M+2 H \int_{M}\langle A \xi, \xi\rangle d M \\
& +\int_{M}\langle\Delta \xi, \xi\rangle d M+2 \int_{\partial M} H^{\partial W}\|\xi\|^{2} d M .
\end{aligned}
$$

Aplying the same arguments to the test functions $\bar{w}_{i}$ we get

$$
\begin{aligned}
\lambda_{\alpha}^{J} \int_{M}\|\xi\|^{2} d M \leq & -4 H^{2} \int_{M}\|\xi\|^{2} d M+2 H \int_{M}\langle A \star \xi, \star \xi\rangle d M \\
& +\int_{M}\langle\Delta \star \xi, \star \xi\rangle d M+2 \int_{\partial M} H^{\partial W}\|\xi\|^{2} d s .
\end{aligned}
$$

Then, summing these last two inequalities and noting that $\langle A \xi, \xi\rangle+$ $\langle A \star \xi, \star \xi\rangle=2 H\|\xi\|^{2}$ we have

$$
\begin{aligned}
\lambda_{\alpha}^{J} \int_{M}\|\xi\|^{2} d M \leq & +2 \int_{\partial M} H^{\partial W}\|\xi\|^{2} d M-2 H^{2} \int_{M}\|\xi\|^{2} d M \\
& +\frac{1}{2} \int_{M}(\langle\Delta \xi, \xi\rangle+\langle\Delta \star \xi, \star \xi\rangle) d M
\end{aligned}
$$

Finally, if $\xi \in \mathcal{L}_{m}^{\Delta}$ we get $\xi=\sum_{i} \alpha_{i} \xi_{i}$ and therefore

$$
\int_{M}\langle\Delta \star \xi, \star \xi\rangle d M=\int_{M}\langle\Delta \xi, \xi\rangle d M=\lambda_{m(\alpha)} \int_{M}\|\xi\|^{2} d M .
$$

Using (4.3) into (4.2) and using the fact that $H^{\partial W} \leq 0$ we obtain

$$
\lambda_{\alpha}^{J} \leq-2 H^{2}+\lambda_{m(\alpha)}^{\Delta},
$$

where $m(\alpha)>6(\alpha-1)$. It concludes the first part of Theorem 1.1.

In order to get the lower bound for the index of $M$ we take $\left\{\phi_{1}, \phi_{2}, \cdots\right\}$ an orthonormal basis of the space $\mathcal{F}$ given by eigenfunctions of the Jacobi operator. From Lemma 3.2 we know that if $\xi \in \mathcal{H}_{T}^{1}(M)$, then the test functions $w_{1}, w_{2}$ and $w_{3}$, belong to $\mathcal{F}$.

We look for vector fields $\xi \in \mathcal{H}_{T}^{1}(M)$ such that for $1 \leq i \leq 3$, the test functions $w_{i}, \bar{w}_{i} \in \mathcal{J}^{\alpha-1}$, for some $\alpha \in \mathbb{N}$, and $\bar{w}_{i} \in \mathcal{F}$. In other words, we have the following system with $6 \alpha-3$ homogeneous linear equations in the variable $\xi$ :

$$
\int_{M} \bar{w}_{i}=\int_{M} w_{i} \phi_{k}=\int_{M} \bar{w}_{i} \phi_{k}=0,
$$

where $1 \leq i \leq 3$ and $1 \leq k \leq \alpha-1$. 
If $\operatorname{dim} \mathcal{H}_{T}^{1}(M)=2 g+k-1>6 \alpha-3$, then the system (4.4) has at least one non trivial solution $\xi \in \mathcal{H}_{T}^{1}(M)$. Following the same steps as above we get

$$
\lambda_{\alpha}^{J} \int_{M}\|\xi\|^{2} \leq-2 H^{2} \int_{M}\|\xi\|^{2}
$$

It implies that $\lambda_{\alpha}^{J}<0$ and then $\operatorname{Ind}_{\mathrm{w}}(M) \geq \alpha$. Since $\alpha$ can be chosen as the largest integer such that $2 g+k-1>6 \alpha-3$ we get

$$
\operatorname{Ind}_{\mathrm{w}}(M) \geq \frac{2 g+k-4}{6} .
$$

\section{REFERENCES}

1. Abdelhamid Ainouz and Rabah Souam, Stable capillary hypersurfaces in a halfspace or a slab, Indiana Univ. Math. J. 65 (2016), no. 3, 813-831. MR 3528820

2. Lucas Ambrozio, Alessandro Carlotto, and Ben Sharp, Index estimates for free boundary minimal hypersurfaces, to appear in Math. Ann. (2017).

3. Maria Athanassenas, A variational problem for constant mean curvature surfaces with free boundary, J. Reine Angew. Math. 377 (1987), 97-107. MR 887402

4. Ezequiel Barbosa, On cmc free-boundary stable hypersurfaces in a euclidean ball, arXiv:1607.00038 [math.DG], to appear in Math. Ann (2016).

5. Marcos Petrúcio Cavalcante and Darlan Ferreira de Oliveira, Lower bounds for the index of compact constant mean curvature surfaces in $\mathbf{R}^{3}$ and $\mathbf{S}^{\mathbf{3}}$, arXiv:1711.07233 [math.DG] (2017).

6. Jaigyoung Choe and Miyuki Koiso, Stable capillary hypersurfaces in a wedge, Pacific J. Math. 280 (2016), no. 1, 1-15. MR 3441213

7. Robert Finn, Equilibrium capillary surfaces, Grundlehren der Mathematischen Wissenschaften [Fundamental Principles of Mathematical Sciences], vol. 284, Springer-Verlag, New York, 1986. MR 816345

8. Ailana Fraser and Richard Schoen, The first Steklov eigenvalue, conformal geometry, and minimal surfaces, Adv. Math. 226 (2011), no. 5, 4011-4030. MR 2770439

9. __ Minimal surfaces and eigenvalue problems, Geometric analysis, mathematical relativity, and nonlinear partial differential equations, Contemp. Math., vol. 599, Amer. Math. Soc., Providence, RI, 2013, pp. 105-121. MR 3202476

10. Sharp eigenvalue bounds and minimal surfaces in the ball, Invent. Math. 203 (2016), no. 3, 823-890. MR 3461367

11. Haizhong Li and Changwei Xiong, Stability of capillary hypersurfaces in a euclidean ball, arXiv:1408.2086 [math.DG] (2014).

12. _ Stability of capillary hypersurfaces with planar boundaries, J. Geom. Anal. 27 (2017), no. 1, 79-94. MR 3606545

13. Rafael López, Constant mean curvature surfaces with boundary, Springer Monographs in Mathematics, Springer, Heidelberg, 2013. MR 3098467

14. _ Capillary surfaces with free boundary in a wedge, Adv. Math. 262 (2014), 476-483. MR 3228434 
15. Johannes C. C. Nitsche, Stationary partitioning of convex bodies, Arch. Rational Mech. Anal. 89 (1985), no. 1, 1-19. MR 784101

16. Ivaldo Nunes, On stable constant mean curvature surfaces with free boundary, Math. Z. 287 (2017), no. 1-2, 473-479. MR 3694683

17. A. Ros, Stability of minimal and constant mean curvature surfaces with free boundary, Mat. Contemp. 35 (2008), 221-240. MR 2584186

18. Antonio Ros, One-sided complete stable minimal surfaces, J. Differential Geom. 74 (2006), no. 1, 69-92. MR 2260928

19. Stable periodic constant mean curvature surfaces and mesoscopic phase separation, Interfaces Free Bound. 9 (2007), no. 3, 355-365. MR 2341847

20. Antonio Ros and Rabah Souam, On stability of capillary surfaces in a ball, Pacific J. Math. 178 (1997), no. 2, 345-361. MR 1447419

21. Antonio Ros and Enaldo Vergasta, Stability for hypersurfaces of constant mean curvature with free boundary, Geom. Dedicata 56 (1995), no. 1, 19-33. MR 1338315

22. Pam Sargent, Index bounds for free boundary minimal surfaces of convex bodies, Proc. Amer. Math. Soc. 145 (2017), no. 6, 2467-2480. MR 3626504

23. Alessandro Savo, Index bounds for minimal hypersurfaces of the sphere, Indiana Univ. Math. J. 59 (2010), no. 3, 823-837. MR 2779062

24. Guofang Wang and Chao Xia, Uniqueness of stable capillary hypersurfaces in a ball, arXiv:1708.06861 [math.DG] (2017).

Instituto DE MATEMÁticA

Universidade Federal de Alagoas

Campus A. C. Simões, BR 104 - Norte, Km 97, 57072-970.

MACEIÓ - AL -BRAZIL

E-mail address: marcos@pos.mat.ufal.br

Departamento de Ciências Exatas

Universidade Estadual de Feira de Santana

Avenida Transnordestina, S/N, Novo Horizonte, 44036-900

FEIRA DE SANTANA - BA - BRAZIL

E-mail address: darlanfdeoliveira@gmail.com 